\title{
The patient's subjective attitude towards screening for breast cancer. Should screening be extended to other forms of cancer?
}

\author{
*T. V. TAYLOR \\ M.D., F.R.C.S.(Ed. \& Eng.) \\ **S. RIMMER \\ M.B., Ch.B., D.M.R.D., F.R.C.R. \\ *A. P. M. Forrest \\ M.D., Ch.M., F.R.C.S. (Ed., Eng., Glas.) \\ Departments of *Clinical Surgery and ** Radiology, Edinburgh Royal Infirmary
}

\begin{abstract}
Summary
A sample of 500 consecutive women without symptoms of breast disease attending a breast screening clinic were investigated regarding their attitude to breast screening and to the extension of the screening programme to other forms of cancer. Attendance at the screening clinic was found to be reassuring by $94.2 \%$, and $96.4 \%$ felt that the screening programme should be extended to include other forms of malignancy. There was a history of either respiratory or alimentary tract symptoms, and of smoking, or a family history of cancer in $41 \%$ of these women. The study shows that Edinburgh women are enthusiastic for breast screening and for its extension to include other forms of cancer.
\end{abstract}

\section{Introduction}

Between 1976 and 19784000 women aged 40-59 years were invited to attend the Edinburgh Breast Screening Clinic. Every woman in this age group on the age-sex registers of 11 general practices was sent an invitation, signed by her general practitioner, to attend the screening clinic. Only women already being treated for breast disease or those too ill to attend were excluded. If there was no response, a second letter was sent 4 to 6 weeks later (British Breast Group, 1978). The study now reported was carried out on a sample of these women to determine their attitude to screening for breast cancer and to an extended programme concerned with the early detection of gastrointestinal tract cancer.

\section{Method}

A sample of 500 consecutive women attending the screening clinic were asked to complete a questionnaire regarding their attitude to breast screening and to extension of the screening programme to other forms of cancer. Only 20 clinic attenders during this study refused to complete the questionnaire. Before answering the questions women were informed that gastrointestinal, lung and breast are the most common types of malignancy in the community and it was suggested that there were investigations which could be performed in the hope of detecting those people at risk of developing cancer of the digestive tract or those who may already have an early tumour. It was pointed out that such primary investigations might involve a double contrast barium meal, a chest $X$-ray, a full blood count, and an examination of a stool sample for occult blood. Should these primary investigations prove abnormal, a gastroscopy, barium enema, sigmoidoscopy or other relevant investigations might prove advisable. It was stressed that the aim of this study was to test opinion in a population already attending a screening clinic.

The first part of the questionnaire related to breast cancer. Patients were asked whether they found the screening clinic worrying or reassuring or both of these. They were also asked whether or not they felt that screening should be extended to other forms of cancer detection. The second part of the questionnaire was designed to determine whether patients had any symptoms suggestive of early gastrointestinal or respiratory disease; whether there was a family history of cancer or pernicious anaemia and whether they had undergone previous abdominal surgery for peptic ulcer. Finally they were asked if they had a chronic cough, whether or not they were smokers and if they had ever smoked more than 10 cigarettes/day for a period of one year.

\section{Results}

Breast screening. Of the 500 women who completed the questionnaire $89.6 \%$ considered that attendance 
at the screening clinic was reassuring; $4.6 \%$ who had been initially worried were later reassured, and only $5.8 \%$ had continual worry. In none of these 500 women was breast cancer detected.

Other forms of screening. An overwhelming majority of women sampled believed that screening should be extended to other forms of cancer $(96.4 \%)$. Only 12 women $(2.4 \%)$ felt that there should be no such extension; 9 of these 12 had not found their experience at the breast-screening clinic reassuring; $1.2 \%$ did not know whether the screening programme should be extended. Conversely, 23 of the 29 women who had continual worry as a result of attending the screening clinic still believed that screening should be extended (Table 1).

TABLE 1. The patients' attitudes to existing screening and its possible extension

\begin{tabular}{lc}
\hline & Patients \% \\
\hline Reassured & 89.6 \\
Worried & 5.8 \\
Worried and later reassured & 4.6 \\
& \\
Screening should be extended & 96.4 \\
Screening should not be extended & 2.4 \\
Don't know & 1.2 \\
\hline
\end{tabular}

Indications for screening for other forms of cancer. The incidence of symptoms referable to the gastrointestinal tract: indigestion, flatulence, abdominal pain, constipation, diarrhoea, vomiting, weight loss and anorexia; or a family history of cancer or pernicious anaemia are shown in Table 2. Smoking habits and respiratory symptoms are shown in Table 3. If these parameters were to be selected as indicating the need for screening for lung and gastrointestinal cancer, then $41 \%$ of women of this age group would be eligible.

TABLE 2. Incidence of gastrointestinal tract symptoms in patients attending for breast screening

\begin{tabular}{lc}
\hline & Patients $\%$ \\
\hline Indigestion & $17 \cdot 6$ \\
Flatulence & $14 \cdot 2$ \\
Abdominal pain & $7 \cdot 8$ \\
Constipation & $12 \cdot 6$ \\
Diarrhoea & $3 \cdot 2$ \\
Vomiting & $2 \cdot 0$ \\
Weight loss & $8 \cdot 2$ \\
Anorexia & $3 \cdot 6$ \\
Family history of malignancy & $28 \cdot 2$ \\
Family history of pernicious anaemia & $7 \cdot 8$ \\
Peptic ulcer surgery & $2 \cdot 2$ \\
\hline
\end{tabular}

TABLE 3. Smoking habits of women attending the screening clinic

\begin{tabular}{|c|c|}
\hline & Patients \% \\
\hline Smokers & $35 \cdot 2$ \\
\hline Smoked $10 /$ day for one year & $34 \cdot 8$ \\
\hline Stopped smoking & $16 \cdot 8$ \\
\hline Chronic cough - Smokers & $3 \cdot 0$ \\
\hline - Non-smokers & $1 \cdot 6$ \\
\hline & \\
\hline 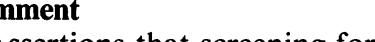 & \\
\hline
\end{tabular}

Assertions that screening for breast cancer cause anxiety and neurosis have not been substantiated iB this particular study. In this sample of woment only $5.8 \%$ were not reassured as a result of attendingr a breast cancer screening clinic. Few of the wome? believed that screening should not be extended to other forms of cancer and, when one considers the incidence of gastrointestinal and lung cancer even i⿺辶卬 females, one could make a case for this. In 1976 i⿺ England and Wales there were 11763 deaths from. breast cancer, 19714 deaths from gastrointestima and 6922 from lung cancers; these 3 malignancie? accounting for $65 \%$ of all female cancer (Repgrt 1976). The attitude of males to such screening programmes is unknown; both gastrointestinas and lung cancer are much more common in the male. Socio-economic factors within the com $\mathbb{\mathbb { D }}$ munity might be more pertinent to the mass screening of males than females and require furthe $\mathrm{B}$ study.

The women screened by the Edinburgh Breas Screening Clinic live in a predominantly middleo class area of southern Edinburgh, although the areas served by the general practices in question include some working-class accommodation, but no. large council estates. From the point of view of thiS socio-economic grouping these women were not perhaps representative of a typical cross-section of the British population, especially as there were fewp immigrants.

Screening for gastrointestinal cancer is mor\& complex than that for cancer of the breast and involves air-contrast barium studies and possible endoscopy. However, limited studies of these methods in symptomatic patients are needed an the role of the haemoccult test in the detection of early gastrointestinal cancer requires full evaluation (Gnauck, 1977; Hastings, 1974).

Primary screening methods must minimize the patient's discomfort, because acceptability of the 
importance and efficiency of screening is vital (Leading Article, 1978). The present study shows that Edinburgh women are enthusiastic for breast screening and for its extension to other forms of cancer.

\section{Acknowledgments}

We thank Dr Anne Scott, Clinical Director of the Edinburgh Breast Screening Clinic, and the Executive Committee of the Edinburgh Breast Screening project, for permission to carry out this study.

\section{References}

British Breast Group (1978) Screening for breast cancer. British Medical Journal, 2, 178.

REPORT of the Office of Population Censuses and Surveys. (1976) Mortality statistics (cause). H.M. Stationery Office, London.

GNAUCK, R. (1977) Dickdarmkarzinom-screening mit haemoccult. Leber Magen Darm, 7 (1), 32.

HASTINGS, J.B. (1974) Mass screening for colo-rectal cancer. American Journal of Cancer, 127, 228.

Leading ARTICle (1978) Screening for gastric cancers in the West. Lancet, i, 1023. 